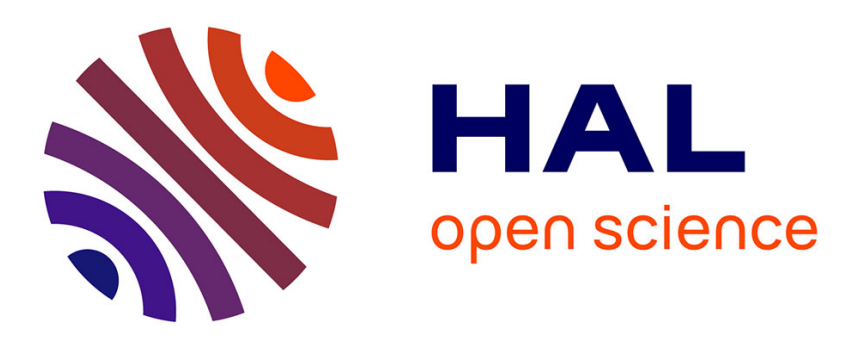

\title{
Contrôle et lutte contre la fraude du patient européen
}

Marion del Sol

\section{To cite this version:}

Marion del Sol. Contrôle et lutte contre la fraude du patient européen. Unionsbürgerschaft und Patientenfreizügigkeit Citoyenneté Européenne et Libre Circulation des Patients EU Citizenship and Free Movement of Patients, Springer, pp.321-336, 2014, 978-3-642-41310-0; 978-3-642-41311-7. 10.1007/978-3-642-41311-7_26. halshs-00956752

\section{HAL Id: halshs-00956752 \\ https://shs.hal.science/halshs-00956752}

Submitted on 13 Jun 2014

HAL is a multi-disciplinary open access archive for the deposit and dissemination of scientific research documents, whether they are published or not. The documents may come from teaching and research institutions in France or abroad, or from public or private research centers.
L'archive ouverte pluridisciplinaire HAL, est destinée au dépôt et à la diffusion de documents scientifiques de niveau recherche, publiés ou non, émanant des établissements d'enseignement et de recherche français ou étrangers, des laboratoires publics ou privés. 
Contrôle et lutte contre la fraude du patient européen - Marion DEL SOL, Professeur de droit, IODE (UMR CNRS 6262-Université de Rennes 1)

Il est des chiffres qui donnent le vertige. Incontestablement, ceux concernant les pertes dues à la fraude et la corruption en matière de soins en font partie. Certaines estimations font en effet état de 56 milliards d'euros perdus annuellement en Europe, ce qui représente près de 80 millions de perte chaque jour et plus de $5 \%$ de l'ensemble des budgets nationaux consacrés à la santé (Gee et alii, 2010).

Mais, derrière ces chiffres, se cachent des réalités multiples qui rendent difficile l'appréhension des phénomènes frauduleux. En effet, plusieurs éléments contribuent à faire de ces phénomènes une terre mal connue. Cela tient à la diversité des agissements frauduleux qui peuvent émaner des nombreuses «parties prenantes » [stakeholders] des systèmes de santé, revêtir des formes variées et être d'ampleur très inégale selon qu'ils sont le fait de patients, de professionnels de santé, d'établissements de santé, de fournisseurs de biens et services médicaux ou encore de laboratoires pharmaceutiques. Si l'on prend l'exemple du seul patient européen, les fraudes peuvent porter sur la réalité des soins (production de faux justificatifs, notamment de fausses factures aux fins de remboursement de soins n'ayant pas été dispensés); sur la nature des soins (production de faux justificatifs aux fins de remboursement de soins reçus à l'étranger mais non pris en charge dans le pays d'affiliation ${ }^{1}$; sur les circonstances des soins (soins présentés comme urgents ou inopinés alors qu'ils correspondent à des soins programmés ${ }^{2}$ ) ; sur la facturation des soins (factures «gonflées » afin de percevoir un remboursement supérieur aux frais engagés) ou encore sur l'identité du patient. Quant au caractère transnational des agissements frauduleux, il est d'évidence facteur d'accroissement des difficultés pour caractériser les fraudes pour l'institution auprès de laquelle la prise en charge des soins est sollicitée puisque, par définition, il y a dissociation entre le pays où les soins (ou présumés soins) sont dispensés et le pays actionné au titre du remboursement.

Si ces réalités se laissent difficilement saisir, s'intéresser à la dimension juridique du contrôle et de la lutte contre la fraude du patient européen nous attire vers une terre inconnue... ou presque. Une des difficultés est en quelque sorte congénitale et tient à l'absence de définition unanimement partagée de la notion de fraude. De façon générale, le recours au terme de fraude se veut «englobant » (fraude lato sensu en quelque sorte) puisqu'il est souvent utilisé pour des comportements de nature, de gravité et d'ampleur pour le moins variées qui vont de l'erreur à la fraude organisée en passant par l'abus et la fraude «isolée »... comportements qui n'impliquent pas nécessairement un élément intentionnel.

L'objectif de l'article n'est pas de lever cette difficulté mais de traiter tant du contrôle que de la lutte contre la fraude en matière de soins transfrontaliers, ce qui permet de s'intéresser à l'ensemble du spectre qui va de l'erreur à la fraude en bande organisée. Par conséquent, le parti est pris de s'intéresser aux mécanismes juridiques qui contribuent «au paiement à bon droit » des soins nécessités par l'état de santé du patient européen, que ces

\footnotetext{
${ }^{1}$ Par exemple, actes de chirurgie plastique «maquillés » en soins a priori remboursables.

${ }^{2}$ Cas de l'utilisation abusive, voire frauduleuse, de la carte européenne d'assurance maladie pour obtenir le remboursement de soins programmés reçus dans un autre État membre alors qu'en principe, la carte permet la prise en charge des soins urgents et non planifiés au bénéfice de personnes tombant malades à l'étranger.
} 
mécanismes aient pour fondement les textes de coordination en matière de sécurité sociale ${ }^{3}$ ou la directive $2011 / 24 / \mathrm{UE}^{4}$.

Autrement dit, l'accent sera porté sur les dispositifs devant permettre que les ressources affectées aux systèmes de santé et d'assurance maladie (spécialement remboursement des soins) soient consacrées à atteindre l'objectif qui leur est assigné (utilisation à bon escient). Par conséquent, dans premier temps, sera mise en évidence la priorité donnée à la logique de paiement à bon droit stricto sensu, priorité qui se manifeste par des mécanismes visant principalement à éviter les erreurs (et abus) de la part des assurés et qui induit l'existence d'outils de contrôle. Puis, dans un second temps, sera abordé le cadre de la lutte contre la fraude à proprement parler dont on peut affirmer qu'il est le parent pauvre des règles applicables au patient européen.

\section{Le paiement à bon droit : $\underline{\text { une priorité }}$}

Que 1'on examine les textes de coordination ou les dispositions de la directive de 2011, transparaît ou est affirmée la volonté de développer (règlement de coordination) ou d'asseoir (directive) la dimension «service aux usagers ». Il convient donc de renforcer l'accessibilité aux droits de ces usagers, notamment au paiement de leurs soins. Cela passe par un droit à information du patient européen, information dont la qualité peut contribuer indirectement à éviter des erreurs (1.1). Mais, dans un cadre transnational, l'effectivité du paiement à bon droit suppose de disposer d'outils dédiés au contrôle et à la lutte contre les erreurs. Dès lors, il conviendra de s'intéresser à l'entraide administrative et aux dispositifs d'échanges d'informations entre les institutions compétentes (1.2).

\subsection{Le droit à information du patient, outil indirect de lutte contre les erreurs}

L'information du patient européen n'est pas conçue à l'identique par les règles de coordination et la directive de 2011. À l'évidence, cela résulte des différences de fondements juridiques de ces textes. Ainsi, la directive reconnaît un droit à information au patient européen afin qu'il puisse faire usage en toute connaissance de cause de la possibilité qui lui est reconnue de se faire soigner dans tout État membre ; l'information joue alors un « rôle pivot dans la promotion de l'accès aux soins transfrontaliers » pour reprendre les termes judicieusement utilisés par Jean-Philippe Lhernould. Les règlements de coordination se préoccupent avant tout des modalités de coppération entre institutions de sécurité sociale dont l'effeicacité doit contribuer à faciliter l'objectif de libre circulation des personnes. Le dispositif d'information est a minima.

\subsubsection{Un dispositif d'information a minima dans le cadre des règles de coordination}

Les règles de coordination des législations de sécurité sociale ont pour objectif de contribuer à la réalisation de l'objectif de libre circulation des personnes, de garantir l'exercice effectif de cette liberté. Par conséquent, le règlement (CE) $n^{\circ} 883 / 2004$ se

\footnotetext{
${ }^{3}$ Règlement $\mathrm{n}^{\circ} 883 / 2004$ du 29 avril 2004 portant sur la coordination des systèmes de sécurité sociale et règlement $\mathrm{n}^{\circ} 987 / 2009 \mathrm{du} 16$ septembre 2009 fixant les modalités d'application du règlement $\mathrm{n}^{\circ} 883 / 2004$

4 Directive 2011/24/UE du 9 mars 2011 relative à l'application des droits des patients en matière de soins transfrontaliers (dont la transposition doit intervenir au plus tard au 25 octobre 2013).
} 
préoccupe avant tout des modalités de coopération entre institutions de sécurité sociale, notamment par l'identification d'organismes de liaison (v. infra).

Certes, l'article 76-4 du règlement dispose que «les institutions et les personnes couvertes par le présent règlement sont tenues à une obligation mutuelle d'information et de coopération pour assurer la bonne application du présent règlement ». Mais il n'est pas affirmé un droit général à information des personnes concernées qui obligerait les institutions de sécurité sociale à mettre en œuvre une démarche systématique d'information. En effet, l'alinéa 2 de l'article 76-4 prévoit des dispositions minimales en la matière : "les institutions, conformément au principe de bonne administration, répondent à toutes les demandes dans un délai raisonnable et communiquent, à cet égard, aux personnes concernées toute information nécessaire pour faire valoir les droits qui leur sont conférés par le présent règlement $»^{5}$.

D'ailleurs, contrairement à la directive de 2011, il n'est pas prévu la création de points de contact nationaux en charge d'une obligation d'information. Il existe «seulement» des centres de liaison. Ainsi, en France, le CLEISS $^{6}$ a notamment pour mission d'être un intermédiaire entre les institutions françaises et étrangères de sécurité sociale et entre celles-ci et les particuliers et les entreprises afin de faciliter, dans le cadre de l'entraide administrative, le règlement de toute difficulté d'ordre juridique ou financier.

$\mathrm{Au}$ regard des personnes concernées, il s'agit surtout de leur permettre de faire valoir leurs droits dans le cadre d'une réglementation complexe. Le considérant 9 du règlement d'application ${ }^{7}$ est explicite à ce sujet : «en raison de la complexité inhérente au domaine de la sécurité sociale, il convient que l'ensemble des institutions des États membres consentent des efforts particuliers en faveur des personnes assurées afin de ne pas pénaliser celles d'entre elles qui n'auraient pas transmis leur demande ou certaines informations à l'institution habilitée à traiter cette demande... ». L'idée directrice est la suivante : lorsqu'une personne sollicite une institution compétente, celle-ci doit prendre en charge la demande de façon efficace en transmettant les informations et délivrant les documents nécessaires dans les délais permettant la « prise en charge » (v. art. 3-4 du règlement d'application).

Toutefois, de façon assez peu visible, les nouveaux règlements semblent vouloir développer le droit à information. En réalité, ce développement ne passe pas par l'affirmation d'une obligation d'information à la charge des institutions nationales (comme cela ressort de la directive de 2011 ; v. infra) ; le droit à information est indirectement envisagé au travers des missions confiées à la commission administrative ${ }^{8}$, commission instituée auprès de la Commission européenne et composée d'un représentant gouvernemental de chaque État membre (art. 71, règlement $\left.\mathrm{n}^{\circ} 883 / 2004\right)$. En effet, l'article 89 du règlement d'application $\mathrm{n}^{\circ}$ 987/2009 - article consacré à l'information - investit la commission administrative de la mission de "préparer les informations nécessaires pour faire connaître aux intéressés leurs droits ainsi que les formalités administratives à accomplir pour les faire valoir ».

\footnotetext{
${ }^{5}$ Afin d'encourager les États membres à être plus « ambitieux » dans le domaine de l'information à destination des assurés, l'article 79 envisage toutefois le financement par la Commission de «toute [autre] action visant à informer les personnes couvertes par le présent règlement et leurs représentants des droits et des obligations découlant du présent règlement, par l'utilisation des moyens les plus appropriés ».

${ }^{6}$ Centre de liaisons européennes et internationales de sécurité sociale.

${ }^{7}$ Règlement (CE) n ${ }^{\circ}$ 987/2009 du Parlement Européen et du Conseil du 16 septembre 2009 fixant les modalités d'application du règlement $(\mathrm{CE}) \mathrm{n}^{\circ} 883 / 2004$ portant sur la coordination des systèmes de sécurité sociale.

${ }^{8}$ Commission administrative pour la coordination des systèmes de sécurité sociale.
} 
L'objectif est de s'assurer, d'une part, de la diffusion de ces informations préférentiellement par leur mise en ligne sur des sites accessibles au public et, d'autre part, de leur mise à jour régulière. Il s'agit également pour la commission administrative de veiller à la qualité du service fourni aux usagers. Pour autant, l'effectivité de cet objectif dépendra de la bonne volonté de chaque État, le règlement n'ayant pas mis à la charge de chacun d'entre eux une obligation d'information dûment exprimée.

\subsubsection{L'affirmation d'un droit à l'information dans le cadre de la directive de 2011}

Bien que la directive soit «relative à l'application des droits des patients en matière de soins de santé transfrontaliers ", sa base juridique est l'article 114 TFUE (établissement et fonctionnement du marché intérieur) comme le justifie le considérant 2. En d'autres termes, si les préoccupations sanitaires sont bien évidemment présentes, la logique "marché intérieur » constitue le fil directeur du texte. Il s'agit de permettre le développement de la libre prestation de service en matière de soins de santé ${ }^{9}$, développement qui dépend en partie de la mobilisation que les personnes intéressées (les patients européens) peuvent faire de leurs droits $^{10}$.

Dès lors, il n'est pas surprenant que la directive cherche à faciliter l'accès aux soins de santé transfrontaliers. Le droit à l'information devient de ce fait un élément important dont le considérant 48 rend compte : "une information adéquate sur tous les aspects essentiels des soins de santé transfrontaliers est nécessaire pour permettre aux patients d'exercer effectivement leurs droits à des soins de santé transfrontaliers ».

Afin que ce véritable droit à information soit effectif, la directive prévoit la création de «points de contact nationaux... pour fournir l'information relative aux principaux aspects des soins de santé transfrontaliers» (considérant 48). Ainsi, 1'article 6-3 précise les obligations d'information pesant sur les points de contact ${ }^{11}$. S'agissant des points de contact de l'État membre d'affiliation (art. 6-4), obligation leur est faite de fournir aux patients des informations concernant leurs droits en matière de soins de santé transfrontaliers, spécialement les conditions de remboursement ${ }^{12}$, les procédures d'accès à ces droits et de détermination de ces droits ${ }^{13}$. Ces informations doivent permettre de «visualiser» la distinction entre les droits au titre des règles de coordination et ceux au titre de la directive. D'ailleurs, le considérant 31 précise que «... lorsque le patient a droit à bénéficier de soins de santé transfrontaliers à la fois au titre de la présente directive et du règlement $(C E)$ $n^{\circ} 883 / 2004$, et que l'application dudit règlement est plus avantageuse pour le patient, l'État membre d'affiliation devrait attirer son attention sur ce point ». Quant aux points de contact de l'État membre de traitement (art. 6-3), il leur appartient de fournir «des informations

\footnotetext{
${ }^{9}$ Considérant $11:$ «... ni leur caractère particulier, ni leur mode d'organisation ou de financement ne saurait faire échapper les soins de santé au principe fondamental de la libre prestation de services... ».

${ }^{10}$ «La raison première de la directive est la codification d'une jurisprudence dont le caractère nécessairement casuistique peut affecter la pleine connaissance, par les patients, des droits qu'ils tirent des libertés de circulation ». Driguez L, Michel V. (2011)

${ }^{11}$ Le texte insiste sur la nécessité que toutes ces informations soient «facilement accessibles et... mises à disposition par des moyens électroniques... » (art. 6-5).

${ }^{12}$ Les critères et conditions de remboursement doivent être connus préalablement (v. art. 7-6 et 7-7 mais aussi considérant 37 sur la possibilité pour les États membres de maintenir des conditions générales, des critères d'admissibilité et des formalités pour recevoir des soins et en obtenir le remboursement : «... il convient que ces conditions générales, ces critères et ces formalités soient connus au préalable... »).

${ }^{13}$ Les informations doivent également concerner les procédures de recours et de réparation dans l'hypothèse où les patients considèreraient que leurs droits ont été méconnus.
} 
relatives aux prestataires de soins de santé » mais aussi «des informations sur le droit des patients, les procédures permettant de porter plainte et les mécanismes de demande de réparation...».

Une meilleure information des patients/assurés contribue à améliorer l'accessibilité aux droits ${ }^{14}$, spécialement dans le cadre de la directive de 2011 qui peut conduire à muer le patient européen «en consommateur averti de soins de santé »(Chrétien, 2011). Cela devrait appeler en retour une sorte «d'éthique de la responsabilité $»^{15}$. Autrement dit, plus l'information du patient sur ses droits est adéquate (accessible, exhaustive et compréhensible), et plus le système peut exiger de ce patient qu'il ne fasse valoir des droits que dans la mesure et les limites fixées par les règles.

Levant l'opacité sur les conditions d'accès aux droits du patient européen, le renforcement de l'information contribue théoriquement à marginaliser les risques d'erreur. Il peut être perçu comme un outil de prévention contre les risques de paiement erroné des soins. Mais il ne s'agit là que d'un outil très indirect de paiement à bon droit... dont l'efficacité est pour le moins incertaine. En termes d'efficacité, on peut attendre bien davantage des mécanismes d'entraide administrative et d'échanges d'informations entre les institutions compétentes.

\subsection{L'entraide administrative et les échanges d'informations, outils dédiés du paiement à bon droit}

Les mécanismes d'échanges d'informations et d'entraide administrative participent d'une démarche coopérative. Ils contribuent à accélérer le paiement des soins transfrontaliers au patient. Ils facilitent également les opérations de contrôle permettant de détecter les situations où le paiement à bon droit est mis à mal ; en effet, dans un contexte transnational, l'efficacité du contrôle implique presque systématiquement de recueillir des informations « localisées » sur un autre territoire que celui de l'institution en charge du remboursement des soins.

\subsubsection{La nécessité et les limites de l'entraide administrative}

Le règlement de coordination $\mathrm{n}^{\circ} 883 / 2004$ consacre un article au thème de la coopération (art. 76). Il y est notamment précisé que «les autorités et les institutions des États membres se prêtent leurs bons offices et se comportent comme s'il s'agissait de l'application de leur propre législation ». Il s'agit donc d'institutionnaliser une véritable entraide administrative.

Il en va de même au titre de la directive de 2011. Si ce texte envisage la coopération sanitaire entre les États membres, il s'intéresse également à l'assistance et à la coopération administrative. Ainsi, l'article 10-1 dispose que «les États membres se prêtent l'assistance mutuelle nécessaire à la mise en ouvre de la présente directive, notamment... par l'échange d'informations... y compris sur les dispositions relatives à la surveillance et à l'assistance mutuelle pour préciser le contenu des factures ». En particulier, en cas de doute sur les informations fournies par les personnes ou encore sur le bien-fondé d'un document ou d'une pièce justificative, voire sur l'exactitude des faits qui sont à la base des mentions y figurant,

\footnotetext{
${ }^{14}$ Pour un exemple d'outil d'information «grand public » à visée pratique en matière d'accès aux soins à l'étranger, voir www.santetransfrontaliere.eu

${ }^{15}$ Propos tenus par J. Vignon lors d'un colloque organisé par l'IPSE en 2008.
} 
l'institution du lieu de séjour ou de résidence doit en principe procéder aux vérifications nécessaires et ce à la demande de l'institution compétente.

Mais l'efficience de cette entraide administrative à l'échelle de l'espace européen est inévitablement problématique. D'ailleurs, le règlement lui-même semble avoir intégré ces difficultés puisqu'il précise que les vérifications sollicitées sont effectuées "pour autant que cela soit possible $»^{16}$. Existent des différences de volonté « coopérative » d'un État membre à l'autre qui peuvent avoir des raisons politiques mais aussi - et sans doute bien davantage des raisons matérielles tenant aux moyens (humains, techniques, ...) que les institutions nationales peuvent mobiliser pour répondre aux demandes d'entraide ${ }^{17}$. On pense notamment aux demandes de contrôle médical qui peuvent être sollicitées sur le fondement de l'article $87-1$ du règlement $n^{\circ} 883 / 2004^{18}$.

Qui plus est, dans le cadre des règles de coordination, le coût du traitement administratif des demandes d'entraide est normalement supporté par l'institution sollicitée ${ }^{19}$ puisque l'article 76-4 du règlement $\mathrm{n}^{\circ}$ 883/2004 dispose que «l'entraide administrative... est en principe gratuite ». Ce principe de gratuité peut constituer un frein pour des institutions pas nécessairement organisées et structurées pour faire face à des demandes d'informations et de renseignements provenant d'autres États membres ${ }^{20}$.

\subsubsection{Les «facilitations " techniques en matière d'entraide administrative}

Afin de fluidifier l'échange d'informations - et in fine de faciliter également les opérations de contrôle du paiement à bon droit-, le règlement de coordination $n^{\circ}$ 883/2004 promeut un recours accru aux nouvelles technologies ${ }^{21}$ et prévoit que la commission administrative peut financer des actions visant à améliorer les échanges d'informations entre les autorités et institutions de sécurité sociale des États membres, en particulier l'échange électronique de données (art. 79).

En réalité, la «facilitation » technique emprunte trois voies dans le cadre du dispositif de coordination. Tout d'abord, est recherché un certain degré de sécurisation «technicojuridique ", l'article 78-4 du règlement $\mathrm{n}^{\circ} 883 / 2004$ postulant comme valide tout document électronique lorsque «le système informatique sur lequel [il] est enregistré comporte les éléments de sécurité nécessaires pour éviter toute altération ou toute communication de

\footnotetext{
${ }^{16}$ Règlement $n^{\circ}$ 987/2009, art. 5-3.

${ }^{17}$ Juridiquement, l'obstacle linguistique n'est en général pas « recevable » puisque «les autorités, institutions et juridictions d'un État membre ne peuvent rejeter les requêtes ou autres documents qui leur sont adressés du fait qu'ils sont rédigés dans une langue officielle d'un autre État membre, qui est reconnue comme langue officielle des institutions de la Communauté, conformément à l'article 290 du traité » (art. 76-7, règlement n 883/2004).

${ }^{18}$ Art. 87-1 : «nonobstant d'autres dispositions, lorsqu'un bénéficiaire ou un demandeur de prestations, ou un membre de sa famille, séjourne ou réside sur le territoire d'un État membre autre que celui où se trouve l'institution débitrice, le contrôle médical est effectué, à la demande de cette institution, par l'institution du lieu de séjour ou de résidence du bénéficiaire conformément aux procédures prévues par la législation que cette institution applique... ».

${ }^{19}$ Par exception, tel n'est pas le cas pour les demandes de contrôle médical dont le coût réel est supporté par l'institution débitrice (art. 87-6).

${ }^{20}$ L'article 76-4 prévoit que la commission administrative peut toutefois établir la nature des dépenses remboursables et les seuils au dessus desquels leur remboursement est prévu.

${ }^{21}$ Art. 78-1 : «les États membres utilisent progressivement les nouvelles technologies pour l'échange, l'accès et le traitement des données requises pour l'application du présent règlement et du règlement d'application. La Commission européenne accorde son soutien aux activités d'intérêt commun à partir du moment où les États membres instaurent ces services de traitement électronique de l'information ».
} 
l'enregistrement ou tout accès non autorisé audit enregistrement ». Ensuite, il est prévu une certaine forme de standardisation/normalisation des documents électroniques donnant lieu à échange puisque, dans le cadre de ses attributions, la commission administrative en fixe la structure, le contenu et le format (art. 4-1 du règlement d'application $n^{\circ}$ 987/2009). L'appellation correspondante est celle de documents électroniques structurés (DES ${ }^{22}$, qui doivent à terme remplacer les formulaires papier de liaison (de type E...).

Enfin, on peut attendre beaucoup de la (future) mise en place d'un système d'échange électronique d'informations sur la sécurité sociale (EESSI). L'article $72 \mathrm{c}$ ) et d) du règlement $\mathrm{n}^{\circ} 883 / 2004$ charge la commission administrative - via la commission technique instituée en son $\operatorname{sein}^{23}$ - de promouvoir et développer la coopération entre les États membres «en modernisant les procédures nécessaires à l'échange d'informations, en particulier en adaptant aux échanges électroniques le flux d'informations entre les institutions ${ }^{24}$. À cet effet, un système informatique d'échange d'information paneuropéen doit voir le jour et se substituer à terme aux échanges papier qui sont encore très utilisés. Les documents électroniques structurés - nouveaux supports d'échange - transiteront par l'EESSI qui sera hébergé par la Commission européenne. Les procédures de vérification et de contrôle seront dès lors substantiellement accélérées et améliorées ${ }^{25}$.

À des degrés divers et selon des modalités différenciées, les règles de coordination et la directive de 2011 instituent un cadre juridique susceptible de limiter les risques de paiement erroné soit en évitant les erreurs par une information préalable suffisante du patient, soit en ayant les moyens de les détecter grâce aux échanges d'informations entre institutions. En comparaison, la lutte contre la fraude apparaît comme le parent pauvre des règles applicables au patient européen.

\section{La lutte contre la fraude : le parent pauvre ?}

En avril 1999, le Conseil a adopté un code de bonne conduite «pour une meilleure coopération entre les autorités des États membres en matière de lutte contre la fraude transnationale aux prestations et aux cotisations de sécurité sociale et le travail non déclaré et concernant la mise à disposition transnationale de travailleurs ». On aurait pu légitimement attendre des textes mettant en jeu la prise en charge des soins transfrontaliers qu'ils contiennent une déclinaison pratique de ce code de bonne conduite adaptée à leur champ. Or, il n'en est rien, les textes représentant en matière de lutte contre la fraude ce que l'on peut appeler un maillon faible (2.1). Il y a davantage à attendre des coopérations interétatiques (2.2) et, dans une autre mesure très différente, de la mise en place de certains réseaux (2.3).

\footnotetext{
${ }^{22}$ ou SED en anglais pour Structured Electronic Documents.

${ }^{23}$ « La commission technique propose à la commission administrative les règles d'architecture commune pour la gestion des services de traitement électronique de l'information, notamment en matière de sécurité et d'utilisation des standards... Elle assure la direction des projets pilotes communautaires d'utilisation de services de traitement électronique de l'information et, pour la partie communautaire, des systèmes opérationnels d'utilisation de ces mêmes services » (art. 73 du règlement $n^{\circ} 883 / 2004$ ).

${ }^{24}$ L'article 4-2 du règlement $n^{\circ} 987 / 2009$ précise que «la transmission de données entre les institutions ou les organismes de liaison s'effectue par voie électronique... dans un cadre sécurisé commun capable de garantir la confidentialité et la protection des échanges de données ».

${ }^{25}$ Le déploiement de l'EESSI est difficile, ce qui a conduit la commission administrative a prolongé de 24 mois la période transitoire avant sa mise en œuvre (décision E3 du 19 oct. 2011). L'EESSI devrait donc être opérationnel au $1^{\mathrm{er}}$ mai 2014.
} 


\subsection{Les textes : le maillon faible}

\subsubsection{La timidité des textes et des décisions y afférentes}

Alors même que le Conseil a adopté, en 1999, un code de bonne conduite "pour une meilleure coopération entre les autorités des États membres en matière de lutte contre la fraude transnationale aux prestations et aux cotisations de sécurité sociale... » et bien que les textes mettent l'accent sur la coopération transfrontalière, ils ne la dédient pas expressément à la lutte contre la fraude. Aucune disposition de la directive de 2011 ni des règlements de coordination n'aborde la question de la lutte contre la fraude. Ces textes sont tout bonnement muets sur cette question... comme si elle ne se posait pas.

Dans le cadre de la coordination, une prise en compte a minima peut cependant être relevée. En effet, au sein de la commission administrative, a été créé un groupe de suivi sur la lutte contre les fraudes et erreurs. La commission administrative elle-même a appelé à la coopération dans ce domaine par une décision du 18 mars 2010 (décision H5) ${ }^{26}$. Elle part du postulat qu'une coopération plus étroite et efficace entre les autorités compétentes et les institutions «est un facteur clé dans la lutte contre les fraudes et les erreurs » (cons. 4) et que l'efficacité passe spécialement par une certaine forme de diligence dans l'échange d'informations sur les changements de législation applicable (cons. 8).

Le contenu - très peu impératif - de la décision H5 traduit assez bien la «timidité » européenne sur cette question de la lutte contre la fraude et les erreurs. Ainsi, les États membres sont invités à désigner un point de contact en matière de fraudes et d'erreurs dont le rôle est a priori modeste. En effet, ce point de contact ne se voit confier aucune mission opérationnelle; il n'est qu'une sorte de collecteur puisque c'est à lui que les autorités compétentes ou institutions peuvent signaler des risques de fraude ou d'abus ou encore des difficultés systématiques à l'origine de retards et d'erreurs (pt. 3).

Sur la base du volontariat, les États membres sont également conviés à établir des rapports retraçant leur expérience et leurs progrès en matière de lutte contre la fraude ${ }^{27}$, rapports devant nourrir les échanges que la commission administrative engage tous les ans sur les questions de coopération dans le domaine des fraudes et des erreurs._Enfin, est exprimé le souhait qu'une assistance diligente et efficace soit mise en œuvre en cas de demande de renseignements visant à lutter contre les fraudes. Ainsi, le point 6 de la décision engage les autorités sollicitées à procéder à «une évaluation minutieuse de la situation juridique avant de rejeter une demande de ce type en invoquant la protection des données »; quant au point 7, il enjoint à l'autorité sollicitée d'apporter son aide au demandeur lorsqu'elle ne traite pas elle-même directement la demande et ce afin que ce demandeur puisse identifier un tiers ad hoc «comme source appropriée d'informations ».

\footnotetext{
${ }^{26}$ Décision $\mathrm{H} 5$ concernant la coopération dans le domaine de la lutte contre les fraudes et les erreurs.

${ }^{27}$ L'annexe 1 de la décision $\mathrm{H} 5$ est une proposition de contenu de ces rapports annuels. Y figure une liste non exhaustive des matières à y aborder : mesures prises tout au long de l'année pour lutter contre les fraudes et les erreurs dans le cadre des dossiers traités au titre des règlements; problèmes spécifiques rencontrés dans l'application des règles de coordination pouvant engendrer à tout le moins des risques de fraudes et d'erreurs ; accords et conventions de coopération bilatérales conclus avec d'autres États membres de l'Union européenne dans le but de lutter contre les fraudes et les erreurs; en ce qui concerne les prestations en nature, mesures prises pour encourager le respect des règles de coordination par les institutions et les prestataires de soins et pour informer les citoyens.
} 


\subsubsection{Les raisons de la timidité des textes}

On peut identifier trois principales raisons ayant conduit le législateur européen à ne pas prévoir un volet explicite de lutte contre la fraude.

Tout d'abord, le constat doit être fait de l'absence de définitions partagées en matière de fraude, ce qui ne permet pas de parler un langage commun, condition préalable pour asseoir des coopérations sur un thème aussi sensible et compliqué que celui de la fraude sociale.

Ensuite, des difficultés systémiques se rencontrant à plusieurs niveaux. Elles résultent, d'une part, des différences - parfois très substantielles - d'organisation et de contenu du système de santé et de protection sociale et, d'autre part, des différences d'organisation et de procédure en matière de contrôle et de lutte ${ }^{28}$.

Enfin, les volonté politiques sont à géométrie variable. On peut constater que peu de pays ont mis en place une organisation structurée de lutte contre la fraude en matière de soins. Pour partie, cette relative «inertie » tient à un manque de volonté politique «d'investir » dans la lutte contre la fraude qui s'explique souvent par une forme d'ignorance de l'ampleur du problème (la fraude étant toujours difficile à chiffrer) et du rapport «coût/bénéfice »des moyens de lutte susceptibles d'être mobilisés (les résultats sont rarement immédiats et tangibles). La lutte contre la fraude sociale en matière de soins transfrontaliers dépend sans nul doute de l'existence préalable d'une volonté politique affichée par l'État membre de lutter contre la fraude sociale dans sa dimension «domestique». Or, cette volonté n'est pas un dénominateur commun aux États membres.

\subsection{Les coopérations interétatiques : le maillon fort}

Une volonté politique nationale peut contribuer à lutter contre la fraude en matière de soins transfrontaliers mais à une échelle qui, elle, restera de dimension limitée... en l'occurrence limitée aux seules factures présentés par des assurés relevant de la sécurité sociale nationale ${ }^{29}$. On peut sans doute attendre bien davantage des coopérations interétatiques.

Malgré sa très grande «timidité » en matière de lutte contre la fraude, le législateur européen a souhaité ouvrir la porte à de telles coopérations dans le cadre des règles de coordination. En effet, l'article 8-2 du règlement de base permet à deux États membres (ou plus) de conclure des conventions bilatérales de sécurité sociale ${ }^{30}$ pour l'application desquelles ils peuvent conclure des arrangements administratifs (visés à l'article 8-2 du règlement $n^{\circ}$ 987/2009). Le règlement d'application fait également place à des accords plus spécifiques conclus entre États membres aux fins de mise en place d'une procédure ad hoc pour l'application des mécanismes de coordination (art. 9-2) ou de certaines de leurs règles (par exemple pour la totalisation des périodes d'activité en matière de retraite).

\footnotetext{
${ }^{28}$ Les unités de contrôle et de lutte ne sont pas nécessairement investies des mêmes missions ; elles ne disposent pas des mêmes moyens, techniques et prérogatives d'investigation (par exemple, en Belgique, l'accès à certaines données est indirect pour les «contrôleurs » car les données pertinentes sont détenues par les mutuelles alors qu'en France, l'accès est direct).

${ }^{29}$ Le partage des enseignements tirés de la politique de contrôle peut toutefois s'avérer utile pour les autres États dans une démarche d'échange de bonnes pratiques... même si l'utilité dépend très largement des moyens que les États acceptent de consacrer à la lutte contre la fraude.

${ }^{30}$ Dans le respect des principes et de l'esprit des règles de coordination.
} 


\subsubsection{L'exemple de l'accord de coopération franco-belge}

Un des (rares) exemples d'accord de cooépration est l'accord franco-belge. Il a pour objet «le développement de la coopération et de l'entraide administrative en matière de sécurité sociale». Cet accord a été signé par les gouvernements des deux pays le 17 novembre 2008 à Bruxelles mais n'a été approuvé par le Parlement français que le 14 mai $2010^{31}$. Cet accord est intéressant en ce qu'il constitue «un dispositif bilatéral novateur... [permettant] une coopération renforcée $\gg^{32}$ :

L'exposé des motifs du projet de loi n 1956 tendant à l'approbation parlementaire, déposé en octobre 2009, souligne l'insuffisance des dispositions relatives à la coopération figurant dans les règles de coordination (en l'occurrence, dans les anciens règlements). À juste titre, il considère que ces dispositions ne permettent pas «la mise en place d'une coopération renforcée, concrète et directe entre les organismes de sécurité sociale ». Or, la France et la Belgique partagent la même volonté de lutter contre la fraude sociale, volonté qui ne «doit pas s'arrêter aux frontières des États » comme le précise l'étude d'impact accompagnant le projet de loi : «il importe... que les contrôles puissent être effectués dans les cas de situations transnationales de la même façon que sont contrôlées les situations nationales conformément à la législation de chacun des États ». Par conséquent, l'accord n'est finalement que le prolongement des politiques «domestiques» de lutte contre la fraude, prolongement qui appelle - pour des raisons d'efficacité - des modalités adaptées au caractère transnational de la fraude contre laquelle il s'agit de lutter.

L'accord institue une obligation de diligence. En vertu d'un principe d'assistance mutuelle qui est réaffirmé ici, l'accord impose aux institutions compétentes de répondre dans un délai de 3 mois maximum à toute demande d'assistance, voire plus rapidement si l'urgence l'exige (art. 5). Il s'agit là d'une obligation générale de coopération et de diligence ${ }^{33}$ puisqu'est posé le principe de la possibilité de recueillir des informations dès lors qu'elles ont pour finalité de garantir une bonne application des droits (art. 12).

Au-delà de l'entraide administrative ponctuelle (en réponse à une demande précise formulée par l'un des institutions compétentes), l'accord prévoit les moyens d'entrer dans une démarche coopérative systématique et opérationnelle. À cet effet, il systématise l'information du partenaire sur toutes les modifications de la réglementation nationale (art. 8), prévoit la transmission et le rapprochement des fichiers de données «en vue de la constatation de fraudes, abus ou erreurs » (art. 6), notamment celles relatives à l'état civil ${ }^{34}$ et met en place un échange systématique d'informations (dans ou hors cadre d'une saisine) sur les suspicions de fraude ou d'erreur (art. 13).

\footnotetext{
${ }^{31}$ Loi n ${ }^{\circ} 2010-487$ du 14 mai 2010 autorisant l'approbation de l'accord entre le Gouvernement de la République française et le Gouvernement du Royaume de Belgique pour le développement de la coopération et de l'entraide administrative en matière de sécurité sociale - JO 15 mai 2010

${ }^{32}$ Rapport fait au nom de la commission des affaires étrangères de l'Assemblée nationale sur le projet de loi n 1956, déc. 2009

33 Au-delà de l'obligation générale de diligence, l'accord envisage de façon spécifique le contrôle de la condition de résidence. Dans ce but, l'institution compétente peut interroger une institution de l'autre État qui est alors tenue de lui répondre pour vérifier la qualité de résident (art. 9).

${ }^{34}$ L'article 6 prend soin de préciser que les opérations de contrôle « respectent le cadre juridique relatif à la protection des données à caractère personnel » et l'article 7 rappelle les dispositions en matière de protection des données à caractère personnel (notamment directive 95/46/CE).
} 
Par ailleurs, l'accord pose le principe d'une assistance mutuelle et de coopération en matière de contrôles (art. 19) et ouvre la possibilité pour les agents d'un État d'être présents lors d'un contrôle organisé par l'autre partenaire et ce en qualité d'observateurs (art. 20). Enfin, des déclinaisons opérationnelles existent. Ainsi, afin de déterminer les modalités de mise en œuvre, l'accord de coopération prévoit la conclusion d'un arrangement administratif conclu entre les autorités compétentes (ministères), arrangement devant notamment détailler les modalités et procédures de transfert des données électroniques (art. 21) ; il ouvre également la possibilité pour les organismes de sécurité sociale eux-mêmes de conclure des conventions de coopération (art. 22) ${ }^{35}$.

\subsubsection{De certaines limites juridiques en matière de coopération interétatique}

L'accord franco-belge peut permettre d'illustrer certains obstacles juridiques à la coopération. La volonté partagée est une chose, la mise en œuvre en est une autre. Elle dépend en grande partie du support juridique choisi pour organiser et encadrer la coopération transnationale. Dans le cas franco-belge, on dispose d'un accord de coopération entre les gouvernements conclu en novembre 2008, d'un projet de loi d'approbation de l'accord déposé devant le Parlement français en octobre 2009, d'une loi « d'approbation » adoptée en mai 2010, d'arrangements administratifs entre ministères et de conventions de coopération entre institutions de sécurité sociale.

Le processus est long en raison de la nécessité de soumettre l'accord de coopération à approbation parlementaire. En effet, pour ce qui concerne la France, cette approbation doit intervenir dès lors que l'on est en présence d'un engagement international relevant de l'article 53 de la Constitution, ce qui est le cas lorsque l'accord comporte des dispositions de nature législative (au sens de l'article 34 de la Constitution) ou est relatif à l'état des personnes. Telle est a priori l'hypothèse des accords de coopération en matière de lutte contre la fraude sociale: ces accords organisant des échanges de données à caractère personnel, il est considéré qu'ils sont - au moins partiellement - relatifs à l'état des personnes. Il en résulte que, lorsque la France est l'une des parties à un accord de coopération tel que celui conclu avec la Belgique, l'entrée en application peut être très largement différée par rapport à la date à laquelle la volonté politique commune s'est concrétisée par la signature d'un accord.

Les parties intéressées (au sens opérationnel du terme) peuvent dès lors avoir tendance à recourir directement ${ }^{36}$ à des arrangements administratifs afin d'échapper à la procédure d'approbation et de permettre une mise en œuvre aussi rapide que possible. L'inconvénient tient à une certaine forme d'incertitude juridique sur la nature et la portée de ces arrangements. Une circulaire de 1997 précise en effet que «ces arrangements constituent une catégorie inconnue du droit international. En conséquence, tout en engageant l'État, ils

\footnotetext{
${ }^{35}$ Dans ce cadre, une convention de coopération a été signée fin 2009 entre la CNAMTS et l'INAMI belge (Institut nationale d'assurance maladie-invalidité). Cette accord institue un cadre réglementé permettant une coopération effective de lutte contre la fraude en matière de soins (notamment en facilitant des échanges rapides de fichiers et d'informations afin d'améliorer les recherches et le contrôle). Exemple extrait du rapport EHCFN sept. 2010 : "The bilateral cooperation agreement (France) and INAMI/RIZIV (Belgium), establishing has already allowed CNAMTS to investigate cases and stop payments. An ex-Frenchman who was living in a home for elderly people in Belgium and who had been claiming to his country of origin, France, for the reimbursement of treatment allegedly given by the home which had a slightly misleading name that could be interpreted as a revalidation centre, had sent invoices and been reimbursed an amount of approximately $€ 9000 /$ month for 30 years !".

${ }^{36}$ C'est-à-dire en dehors de l'application d'un accord international de coopération.
} 
présentent l'inconvénient de n'offrir aucune sécurité quant à leur exécution par l'autre partie. Il ne faut donc recourir à ces instruments que dans des circonstances particulières, pour compléter ou préciser un accord existant, ou, à la rigueur, pour organiser une coopération administrative de portée limitée. Dans tous les cas, les ministres ne peuvent s'engager que dans la stricte limite de leurs attributions et la compétence de la partie étrangère doit être vérifiée autant que possible $»^{37}$.

\subsection{Les initiatives en marge du cadre institutionnel : European Healthcare Fraud and Corruption Network (HEFCN)}

En 2005, suite à une initiative d'un ancien responsable du NHS britannique, a été créée une association dont le but est de combattre la fraude et la corruption dans le domaine de la santé en Europe $^{38}$. Il s'agit en réalité d'un réseau ayant vocation à regrouper des membres tant des autorités gouvernementales (telles que des ministères de la santé) que des institutions de sécurité sociale ou encore des assureurs privés, voire des unités spécifiquement dédiées à la lutte contre la fraude.

Le EHFCN est un réseau bien davantage qu'une structure. Son ambition générale est de participer à l'amélioration de la prise en charge sanitaire en Europe en réduisant les pertes dues à la fois à la fraude et à la corruption. Dès lors, il s'agit d'aider les membres du réseau à développer au mieux leur activité dans le domaine de la santé grâce à la diffusion d'une culture anti-fraude ${ }^{39}$ et de bonnes pratiques de lutte contre la fraude. Cependant, le nombre de membres de l'EHFCN (18 provenant de 13 pays) est réduit par rapport aux ambitions initiales, ce qui en soi est un facteur qui limite les possibilités de faire avancer les choses.

\subsubsection{Les pratiques au sein du réseau en matière de soins transfrontaliers}

La lutte contre les fraudes en matière de soins transfrontaliers constitue une des priorités du réseau mais qui se heurte à la très grande autonomie des systèmes nationaux. Sur cette thématique, on est très loin d'une coopération organisée et structurée.

Au niveau du réseau lui-même, il existe des pratiques de coopération informelle puisque la plateforme est utilisée par les membres du réseau à des fins d'échange de données ou pour solliciter des réponses. Pour l'instant, il n'existe qu'un exemple d'une pratique de coopération «formelle » : l'accord bilatéral de coopération qui a été signé en 2009 entre la CNAMTS et l'INAMI/RIZIV (Institut national d'assurance maladie-invalidité, Belgique) ${ }^{40}$, accord qui institue un cadre réglementé permettant une coopération effective de lutte contre la fraude en matière de soins, notamment en facilitant des échanges rapides de fichiers et d'informations afin d'améliorer les recherches et le contrôle.

Par ailleurs, des liens existent entre l'EHFCN et la Commission européenne qui est intéressée pour voir « où le problème se situe » dans le domaine des soins transfrontaliers. Cependant, la « récolte » est maigre car les données sont souvent détenues par des organismes non membres du réseau.

\footnotetext{
${ }^{37}$ Circulaire du 30 mai 1997 relative à l'élaboration et à la conclusion des accords internationaux, JORF $\mathrm{n}^{\circ} 125$ du 31 mai 1997, p. 8415

${ }^{38}$ Seule structure dédiée à cet objectif au niveau européen.

${ }^{39}$ Selon les propres termes du président actuel de l'EHFCN (Paul Vincke), il convient « d'élever la conscience du problème de la fraude».

${ }^{40}$ Devraient également voir le jour des accords Belgique/Pays-Bas et France/Allemagne.
} 


\subsubsection{Les perspectives de l'EHFCN : vers une réorientation de l'action du réseau.}

l'EHFCN.

Afin de agner en efficacité, deux principales pistes d'évolution sont identifiées par

D'une part, le réseau va sans doute mettre davantage en avant la lutte contre le gaspillage ${ }^{41}$ qui est une notion plus intéressante parce que plus facile à comprendre de tous les partenaires et moins négativement connotée que la fraude (qui a une connotation répressive). Il va aussi s'engager davantage sur le terrain de lutte contre la corruption (notamment économie informelle) qui touche fortement certains pays de l'est et du sud de l'Europe (par exemple, en Grèce dans le secteur des médicaments).

D'autre part, le EHFCN envisage de développer un partenariat public/privé car les assureurs privés ont une volonté affirmée d'installer des systèmes anti-fraude et sont beaucoup plus avancés que les acteurs publics sur ces questions. Par conséquent, le réseau va en quelque sorte «cibler » les assureurs privés dans les années à venir car ils sont plus coopératifs et présentent l'avantage de tous parler le même langage quel que soit le pays dans lequel ils développent leur activité... ce qui n'est pas le cas des acteurs publics en raison des grandes différences de configuration des systèmes de santé et de protection sociale.

Paul Vincke, l'actuel président de l'EHFCN, utilise une expression forte lorsqu'il parle de la fraude en matière de soins : «healthcare fraud is a killer». Dans une Europe élargie, il convient sans nul doute contrecarrer les agissements de ce « criminel ». Mais, dans une Europe élargie, n'est-ce pas là une véritable gageure ?

\section{Bibliographie}

Chrétien S. (2011), Droit européen des patients en matière de soins de santé transfrontaliers, Éd. Les études hospitalières, coll. Essentiel, nov., 144 p.

De la Rosa S., (2012), Quels droits pour les patients en mobilité ? À propos de la directive sur les droits des patients en matière de soins transfrontaliers, Revue française des affaires sociales, $\mathrm{n}^{\circ} 1$, pp. 108-129.

Driguez L., Michel V., (2011), La directive 2011/24/UE relative à l'application des droits des patients en matière de soins de santé transfrontaliers : progrès pour la santé ou le marché ?, Revue Europe, oct., pp. 4-10

Fasquelle D., (2010), Les enjeux et risques des soins transfrontaliers, Gazette du Palais, $\mathrm{n}^{\circ} 303$, oct., p. 16

Filhon G. et al., (2012-a), Mobilité des patients et coordination européenne, Revue française des affaires social, $\mathrm{n}^{\circ} 1$, pp. 103-107

Filhon G. et al., (2012-b), La mobilité des patients dans l'Union européenne : les enjeux de la prise de décision, Pratiques et Organisations des Soins, vol. 43, oct.-déc., pp. 285-294 ${ }^{41}$ Par exemple, surconsommation de soins, inefficience des procédures administratives, inefficacité des
trajectoires de santé. 
Gee J., Button M., Brooks G., (2010), The financial cost of healthcare fraud, University of Portsmouth, MacIntyre Hudson, EHFCN

Lhernould J.-P., (2008), La libre circulation du patient en Europe, Les Petites Affiches, $\mathrm{n}^{\circ} 120$, p. 5

Mavridis P., (2008), La lbre circulation des aptients : la boucle est bouclée ?, Les Petites Affiches, $\mathrm{n}^{\circ} 119, \mathrm{p} .4$ 